\title{
Fabrication of Large-Size Alumina by Pressure-Vacuum Hybrid Slip Casting
}

\author{
Kyeong-Sik Cho ${ }^{\dagger}$ and Seung Yeul Lee* \\ School of Advanced Materials and System Engineering, Kumoh National Institute of Technology, \\ Gyungbuk 730-701, Republic of Korea \\ *Dicera Co. Ltd, Gyungbuk 730-853, Republic of Korea
}

(Received Septembe 30, 2013; Revised October 28, 2013; Accepted October 29, 2013)

\begin{abstract}
The size of various alumina ceramics used in the semiconductor and display industries must be increased to increase the size of wafers and panels. In this research, large alumina ceramics were fabricated by pressure-vacuum hybrid slip casting (PVHSC) employing a commercial powder, followed by sintering in a furnace. In the framework of the PVHSC method, the consolidation occurs not only by compression of the slip in the casting room but also by suction of the dispersion medium from the casting room. When sintered at $1650^{\circ} \mathrm{C}$ for $4 \mathrm{~h}$, the fabricated large-size alumina $\left(1,550 \times 300 \times 30 \mathrm{~mm}^{3}\right)$ exhibited a dense microstructure corresponding to more than $99.2 \%$ of the theoretical density and a high purity of $99.79 \%$. The flexural and compressive strengths of the alumina plate were greater than $340 \mathrm{MPa}$ and 2,600 $\mathrm{MPa}$, respectively.
\end{abstract}

Key words : Alumina, Slip casting, Microstructure, Strength

\section{Introduction}

$\mathrm{T}$ he size of alumina ceramics used in the manufacturing processes of the semiconductor and display industries needs to be increased in order to achieve larger wafers and panels. ${ }^{1)}$ Large-size alumina is a promising inorganic material in those fields of application in which the speed of technical innovations and changes in size are very high.

Conventional forming methods for preparing large alumina ceramics, such as cold isostatic pressing, gypsum-mold slip casting, and filter pressing, have several disadvantages. The cold isostatic pressing method is not suitable for the successful production of large industrial products because of limitations arising from inhomogeneous packing and insufficient equipment chamber size. ${ }^{2-4)}$

In gypsum-mold slip casting, differential shrinkage can be caused by a relative green density gradient that can be induced in the molds during water removal. Furthermore, the used molds have a low toughness, so they can fracture easily. Therefore, a large mold inventory must be maintained to ensure constant production quality and to avoid any gradient of green density along the consolidation direction, induced by unidirectional casting. ${ }^{5-9)}$

Filter pressing is another forming method for large ceramics by fluid removal from concentrated colloidal slurries. A green body is formed on a porous filter mold by the applica-

\footnotetext{
${ }^{\dagger}$ Corresponding author : Kyeong-Sik Cho

E-mail : kscho@kumoh.ac.kr

Tel : +82-54-478-7737 Fax : +82-54-478-7764
}

tion of external mechanical pressure: the solid and dispersion medium are separated by the filter. However, when the plate is large and thick, the application of homogeneous pressure over the entire volume is difficult. ${ }^{10-15)}$

These problems could be avoided by employing pressurevacuum hybrid slip casting (PVHSC). In this method, unlike in the usual colloidal routes, the consolidation by hybrid pressure casting occurs not only in the form of a stepwise compression of the slip into the casting room but also by suction of the dispersion medium from the casting room. The driving force for slip casting in this process is a gradual pressure difference either induced by applying pressure in a stepwise manner to force the liquids through the filter (pressure slip casting) or by using a vacuum on the downstream side of the filter where the filtrate is to be collected (vacuum slip casting). ${ }^{16-18)}$ Many studies have shown that in slip casting or filter pressing, the conventional practice of applying static pressure during the casting process may induce a density gradient in the produced body, which in turn results in cracks and distortions after the firing process. ${ }^{19-23)}$ However, in the present study, stepwise pressure loading during slip casting minimized such defects in the final, large-size alumina products.

\section{Experimental Procedure}

A commercial $\mathrm{Al}_{2} \mathrm{O}_{3}$ powder (mean size: $0.5 \mu \mathrm{m}$ ) was used as a raw material. ${ }^{18)}$ Alumina powders at a concentration of $40 \mathrm{vol} \%$ were dispersed in ion-exchanged water including the dispersant APC (ammonium polycarbonate), plasticizer PEG-400 (polyethylene glycol), and binder PVA-205 (polyvi- 


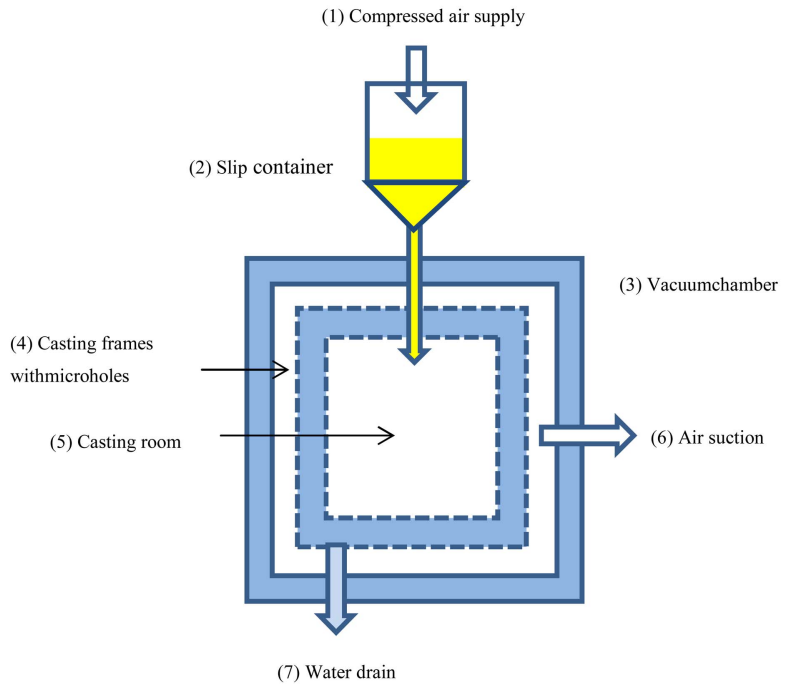

(a)

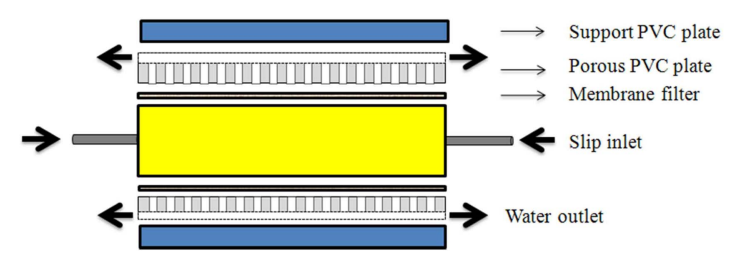

(b)

Fig. 1. Schematic diagrams of (a) the pressure-vacuum hybrid slip casting (PVHSC) apparatus and (b) the casting mold.

nyl alcohol) to form slurries. The dispersant content was $0.4 \mathrm{wt} \%$ in the slip, and that of other components amounted to $1.0 \mathrm{wt} \%$, respectively. The slurry for PVHSC was ballmilled with $\mathrm{Al}_{2} \mathrm{O}_{3}$ media and vacuum-treated for de-airing. The viscosity of the slurry of the alumina-water system was $176 \mathrm{cP}^{18)}$

A PVC casting mold was used to produce a large-size alumina compact $\left(1,855 \times 360 \times 35.5 \mathrm{~mm}^{3}\right)$ by PVHSC, as shown in Fig. 1. The PVHSC processing step was performed in a plate die using a polypropylene fiber-woven membrane filter supported on a porous PVC plate with pores having a diameter of $2-\mathrm{mm}$. The slip inlet load was applied in five

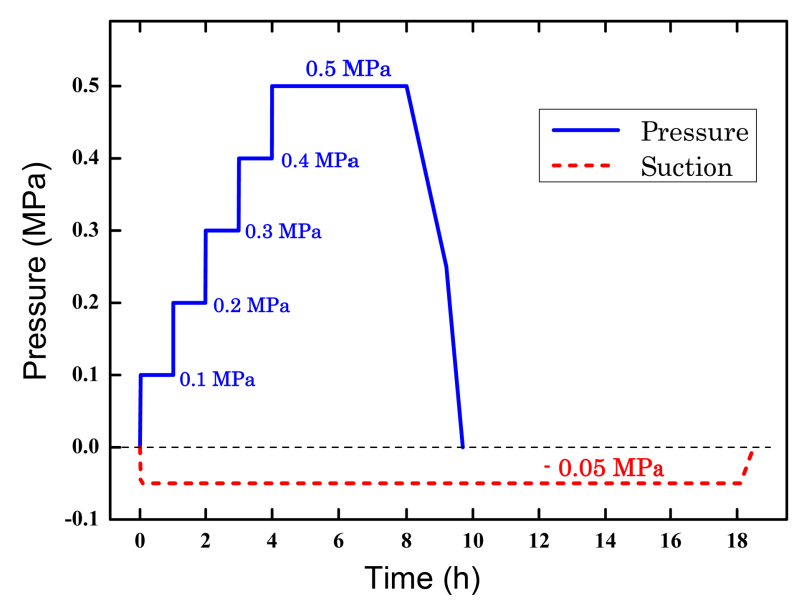

Fig. 2. Applied pressure and suction schedules for pressurevacuum hybrid slip casting.

steps up to $0.5 \mathrm{MPa}$ by a compressor, and air was pumped continuously with a pressure of $-0.05 \mathrm{MPa}$ applied on the downstream side of the filter to enhance the removal of the filtered water, as shown in Fig. 2. After setting the apparatus as in Fig. 3, one forming process cycle took about $18 \mathrm{~h}$.

Plate filter cakes were then allowed to dry at a temperature of 30 with $80 \%$ relative humidity to ensure a slow drying rate. As-formed specimens for measurements were prepared by cutting the alumina block into small pieces with different sections and grinding them carefully with a fine SiC paper, as shown in Fig. 4(a). Green densities were determined geometrically.

Sintering was carried out in an electrical furnace with $\mathrm{MoSi}_{2}$ heating elements in air. During the sintering process, the specimens were heated at a heating rate of $10^{\circ} \mathrm{C} / \mathrm{min}$ up to the maximum temperature $1650^{\circ} \mathrm{C}$ with an isothermal dwell time of $4 \mathrm{~h}$ along with intermediate soaking periods at 350,600 , and $900^{\circ} \mathrm{C}$ to remove organic residues. ${ }^{14)}$ The furnace was switched off for cooling and the samples were taken after the furnace temperature reached below $100^{\circ} \mathrm{C}$.

The density of the sintered specimens was determined by employing the Archimedes method. Microstructure observations were performed using a FE-SEM (JSM-6701F, JEOL, Japan). ICP-AES (Optima 4300, Perkin Elmer, USA) was used to determine trace impurities $\left(\mathrm{MgO}, \mathrm{Na}_{2} \mathrm{O}, \mathrm{SiO}_{2}\right.$ and

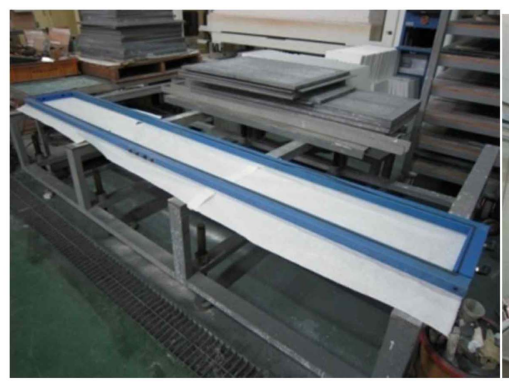

(a)

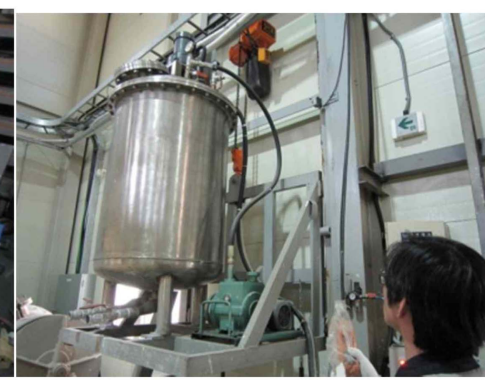

(b)

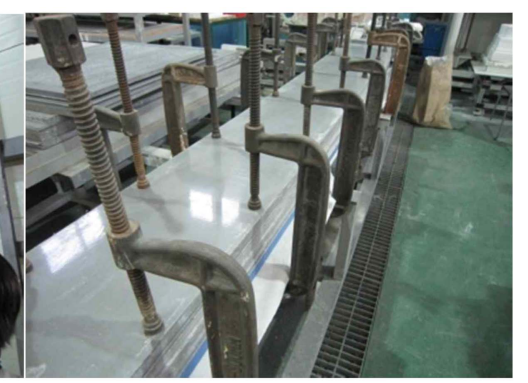

(c)

Fig. 3. Photographs of the pressure-vacuum hybrid slip casting process for fabricating large-size alumina: (a) casting frame, (b) slip inlet, and (c) setting apparatus. 


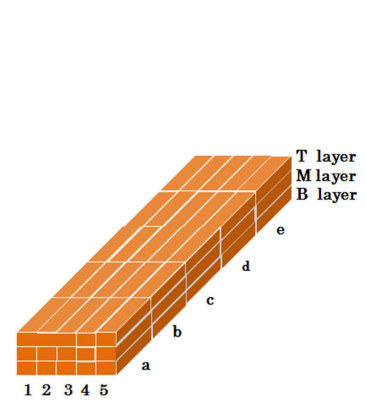

(a)

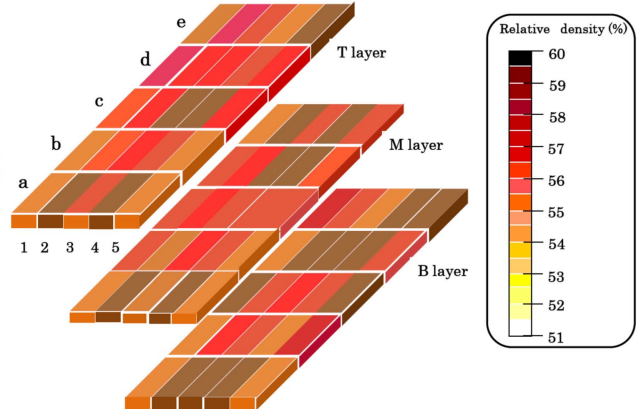

(b)

Fig. 4. Partial density distribution along the thickness of the alumina green bodies manufactured by pressure-vacuum hybrid slip casting: (a) numbering scheme for each section in the body and (b) density distribution of cast alumina layers.

$\mathrm{Fe}_{2} \mathrm{O}_{3}$ ) in the sintered and powder samples. The mechanical properties were evaluated by flexural and compressive strengths. At least six samples were acquired from each section of the different layers within the sintered body. Flexural strength tests were performed at room temperature using a three-point banding method with a span of $20 \mathrm{~mm}$ and a crosshead speed of $0.5 \mathrm{~mm} / \mathrm{min}$. Test bars were cut from each section part within the sintered body and ground to a dimension of $3 \times 4 \times 35 \mathrm{~mm}$. Compressive tests were conducted by applying a uniaxial compressive load (Model 4468, Instron Corporation, USA) on the cylindrical specimen $(\phi 4 \times 12 \mathrm{~mm})$. Samples were acquired from each section part within the sintered body and those ends were enough plane geometry, parallel and perpendicular to the axis of the specimen in accordance with ASTM C $773 .^{24)}$

\section{Results and Discussion}

Well-formed large-size alumina green bodies $(1,855 \times 360 \times$ $35.5 \mathrm{~mm}^{3}$ ) were fabricated by PVHSC employing appropriate casting and drying conditions. A stepwise increase of the pressure, instead of a static pressure load, during slip casting and a slow drying of the cast bodies at high humidity conditions in an unconstrained manner were important for minimizing the appearance of defects in the final, large-size alumina products. ${ }^{16-18)}$

In slip casting, the porous structure of the mold provides suction pressure ranging from 0.1 to $0.2 \mathrm{MPa}$ as the microstructure of the gypsum-mold. ${ }^{15)}$ The magnitude of applied pressure for pressure filtration is related to the equipment and can be as high as $80 \mathrm{MPa}$ when stainless steel equipment is used. ${ }^{10)}$ When the slip is loaded into the mold, water is sucked out by the capillarity, leaving a layer of cake on the slip-mold interface. The kinetics of the casting process can be explained as Darcy's differential equation for fluid flow through porous media. ${ }^{5,6,15)}$ An increase in cake thickness and a consequent decrease in cake permeability with casting time has already been reported for pressure slip cast $\mathrm{SiC}$ bodies. ${ }^{7)}$ The less permeable microstructures were attributed to an improved particle rearrangement enabled by the slowing down of the casting process as the cake thick- ness increased. The pressure drop across the consolidated layer (cake thickness) increases with an increasing volume and the casting time of the filtrate when the applied pressure is constant. ${ }^{5,15)}$ Therefore, the structure is less consolidated at the cake surface and becomes more compact as the mold is approached. To control the kinetics of the casting process, applying a stepwise increase of the slip inlet pressure during slip casting can minimize this deviation of pressure drop. ${ }^{8,25,26)}$ Therefore, the resultant microstructure of the cake produced by a stepwise pressure loading will be more homogenous than the one of static pressure loading. In this work, the slip inlet loading was applied in five steps up to a maximum (0.5 MPa) and continuously water pumped to achieve a pressure of $-0.05 \mathrm{MPa}$, which turned out to be the best casting conditions for the PVHSC process.

The appearance of cracks or a distorted surface in largesize ceramics is a phenomenon that limits the maximum rate at which the drying operation can take place. During fast drying, a moisture gradient is rapidly established, which leads to greater free shrinkage (shrinkage that would occur if there were no mechanical stresses). This free shrinkage gradient induces a stress profile in the plate which, in turn, results in the formation of a compression layer in the inner region of the plate and tension layers near the upper and lower surfaces, which result in cracking and distortions. ${ }^{27-29)}$ Therefore, we employed a slow drying rate with high humid conditions (80\% relative humidity) in this work.

Figure 4 and Table 1 reveal the green density from the bottom to the top of the alumina cast body. The density of each layer from top to bottom was as follows: the top layer, $58.7 \%$; the middle layer, 58.7\%; and the bottom layer, $59.2 \%$. A very uniform density distribution along the direction of thickness of the cast body was measured. The green density for the cast alumina produced by PVHSC was $58.5 \%$ of the theoretical value. Further, the standard deviations of the densities for pieces of cakes were 1.0-1.3. Because of this small level in density deviation of the cast bodies, it is difficult to generate deformations or cracking. Deformation arises as varying parts of the body shrink to different extents which, in turn, can be related to spatial variations 
Table 1.Relative Density of the Different Layers of Alumina Green Body, Fabricated by Pressure-Vacuum Hybrid Slip Casting

\begin{tabular}{cccc}
\hline Layer & Mean (\%) & Standard deviation (\%) & Total body mean (\%) \\
Top & 58.7 & 1.3 & 58.9 \\
Middle & 58.7 & 1.1 & \\
Bottom & 59.2 & 1.0 & \\
\hline
\end{tabular}

Table 2. Chemical Compositions of the Starting Powders and Sintered Bodies

\begin{tabular}{|c|c|c|c|c|c|c|}
\hline \multirow{2}{*}{$\begin{array}{l}\text { Chemical } \\
\text { composition }\end{array}$} & \multicolumn{2}{|c|}{ Starting powders (wt\%) } & \multicolumn{4}{|c|}{ Sintered body (wt\%) } \\
\hline & Supplier data ${ }^{\dagger}$ & Analysis & Top part & Middle part & Bottom part & Mean \\
\hline $\mathrm{Al}_{2} \mathrm{O}_{3}$ & 99.85 & 99.83 & 99.79 & 99.80 & 99.79 & 99.79 \\
\hline $\mathrm{MgO}$ & 0.06 & 0.06 & 0.07 & 0.07 & 0.07 & 0.07 \\
\hline $\mathrm{Na}_{2} \mathrm{O}$ & 0.05 & 0.06 & 0.05 & 0.06 & 0.06 & 0.06 \\
\hline $\mathrm{SiO}_{2}$ & 0.03 & 0.04 & 0.05 & 0.04 & 0.06 & 0.05 \\
\hline $\mathrm{Fe}_{2} \mathrm{O}_{3}$ & 0.01 & 0.01 & 0.04 & 0.03 & 0.04 & 0.04 \\
\hline
\end{tabular}

${ }^{\dagger}$ Sumitomo Chemical Co., Japan
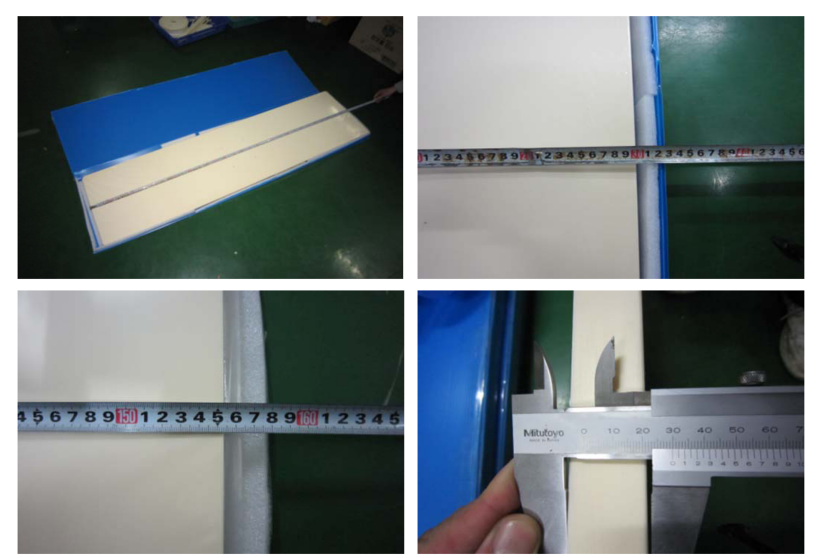

Fig. 5. Photographs of the large-size alumina specimen $(1,550 \times$ $300 \times 30 \mathrm{~mm}^{3}$ ), fabricated by pressure-vacuum hybrid slip casting.

in density within the component. ${ }^{26,29)}$

Figure 5 shows photographs of the large-size alumina specimen that was prepared by PVHSC from aqueous slurry and sintered at $1650^{\circ} \mathrm{C}$ for $4 \mathrm{~h}$ in air. Linear shrinkages of the alumina plate $\left(1,555 \times 300 \times 30 \mathrm{~mm}^{3}\right)$ were about $16.4 \%, 16.1 \%$, and $15.5 \%$ along the length, width and thickness directions, respectively. Relative densities of all sectioned samples were nearly identical. The sintered density was greater than $99.2 \%$ of the theoretical value within a standard deviation of $1.8 \%$, as shown in Fig. 6 .

Figure 7 illustrates the final microstructure of the different layers of the alumina sample. The microstructure of the sintered bodies was examined by FE-SEM, where a fine and uniform microstructure with an average grain size of several micrometers was observed. No systematic changes in the sintered microstructure were observed across the height or cross-section of the sample. Nearly identical microstructures were found in the top, middle and bottom layers. Concerning the grain-size distribution, no significant difference between the layers was observed. This suggests that it is mainly the high degree of homogeneity in the green body

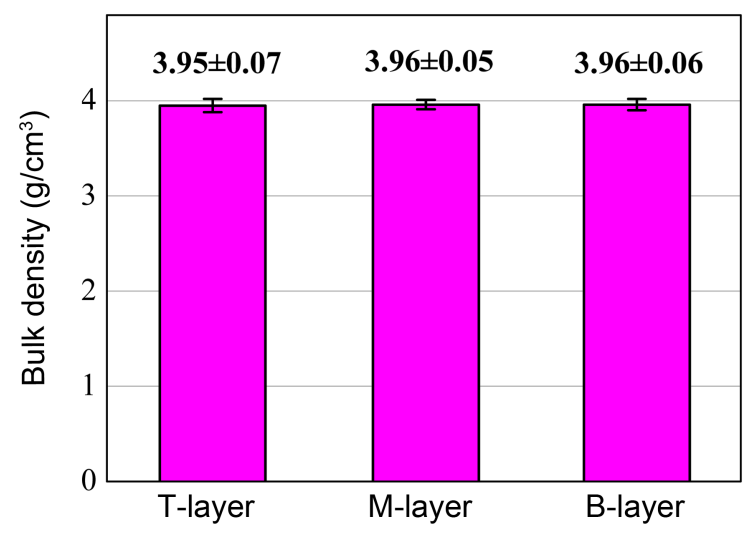

Fig. 6. Relative density of the different layers of the alumina sintered body produced by pressure-vacuum hybrid slip casting. Columns and bars represent mean values and corresponding standard deviations, respectively.

that affects the final microstructure of this dense ceramics.

The chemical compositions of the starting powders and sintered alumina were analyzed by ICP-AES. Data according to manufacturer's declarations are included in Table 2. The purity level from the bottom to the top layers of the sintered alumina and starting powders had almost the same high (> $99.79 \mathrm{wt} \%$ for the sintered body and $>99.83 \mathrm{wt} \%$ for the powders). The impurities in alumina significantly affect their chemical and physical properties. A minor impurity causes development of an inhomogeneous microstructure and reduces the mechanical, chemical and electrical properties of alumina. ${ }^{30)} \mathrm{A}$ high level of such impurities also limits the application of the manufacturing process of the semiconductor and display industries. Therefore, caution is necessary to avoid incorporating impurities during processing steps, such as ball milling, forming and firing.

Figure 8 summarizes the mechanical strengths of the different layers of the large-size alumina specimen. It is obvious that flexural strength and compressive strengths do not vary significantly among the different layers. The average flexural and compressive strengths obtained for the samples 


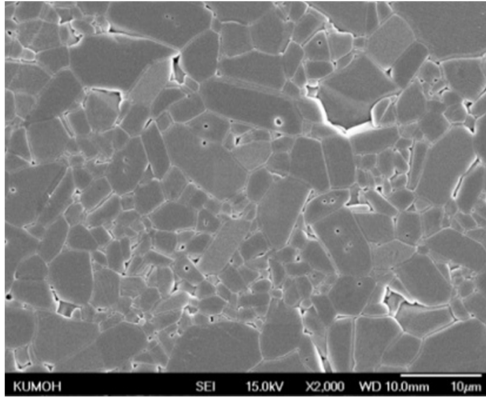

(a)

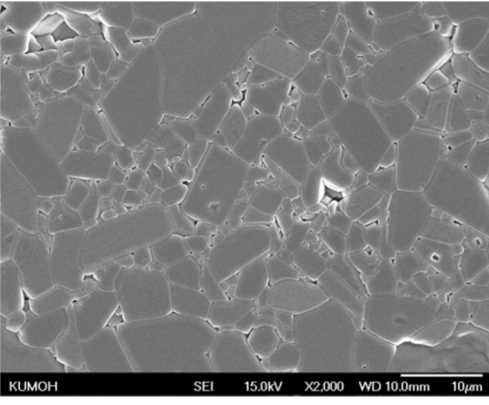

(b)

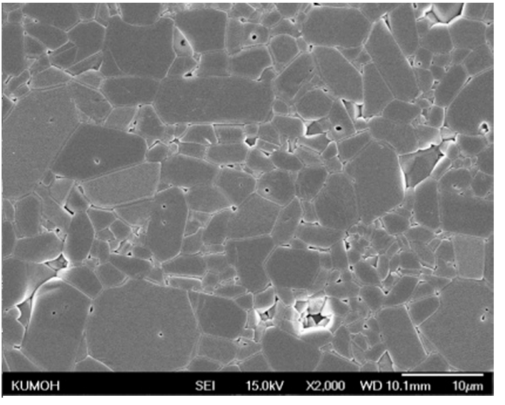

(c)

Fig. 7. Typical SEM microstructures of each layer of the alumina sintered body produced by pressure-vacuum hybrid slip casting: (a) top layer, (b) middle layer, and (c) bottom layer.

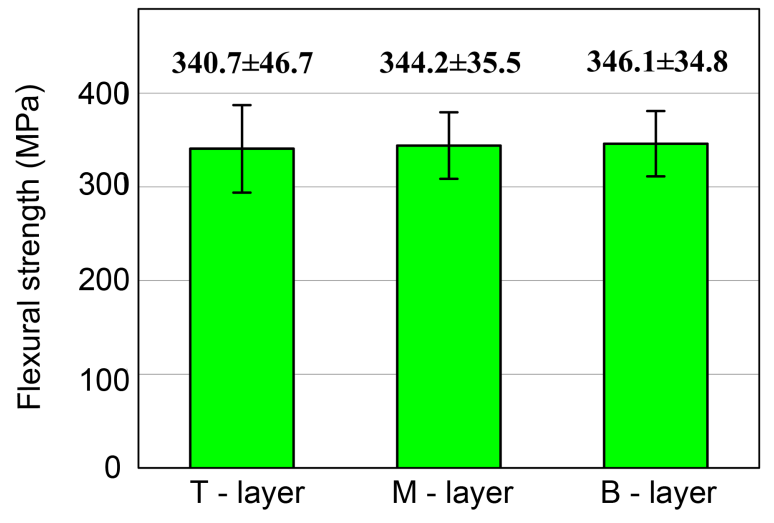

(a)

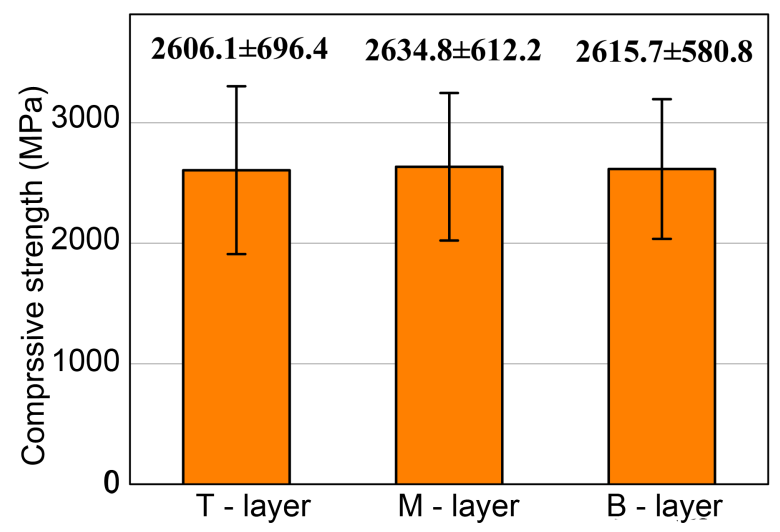

(b)

Fig. 8. Flexural strength (a) and compressive strength (b) of the different layers of the alumina sintered body produced by pressure-vacuum hybrid slip casting. Columns and bars represent mean values and corresponding standard deviations, respectively.

sintered at $1650^{\circ} \mathrm{C}$ were $344 \mathrm{MPa}$ and $2,619 \mathrm{MPa}$, respectively. The range of those values for the pure and finegrained alumina ceramics were 280-420 $\mathrm{MPa}$ and 2,200$2,600 \mathrm{MPa}$, respectively. ${ }^{31,32)}$ These properties will very suitably support PVHSC in producing large-size alumina ceramics.
This method enabled us to fabricate an alumina plate with dimensions of $1,550 \times 300 \times 30 \times \mathrm{mm}^{3}$ without cracks and with a homogeneous density. The possibility of extending this method to increase the product size or fabricate other ceramic materials seems to be promising.

We prepared a large-size alumina plate without cracks and with a homogeneous density by pressure-vacuum hybrid slip casting (PVHSC) from an aqueous slip consisting of $40 \mathrm{vol} \%$ solid and organic additives.

\section{Conclusions}

We prepared a large-size alumina plate without cracks and with a homogeneous density by pressure-vacuum hybrid slip casting (PVHSC) from an aqueous slip consisting of $40 \mathrm{vol} \%$ solid and organic additives. Well-formed large-size green bodies were fabricated by the PVHSC method by applying a stepwise increase of pressure for loading up to $0.5 \mathrm{MPa}$, combined with suction at a pressure of $0.05 \mathrm{MPa}$ using an alumina slip. The green bodies were dried at $30^{\circ} \mathrm{C}$ with $80 \%$ relative humidity. There were no signs of cracking or distortions in the green products. Densities of the green bodies were $58.5 \%$ of the theoretical value and represented a homogeneous distribution along the thickness. When sintered at $1650^{\circ} \mathrm{C}$ for $4 \mathrm{~h}$, the large-size $\left(1,550 \times 300 \times 30 \mathrm{~mm}^{3}\right)$ alumina specimen reached its full density ( $>99.2 \%$ of the relative density). The flexural and compressive strengths of the alumina plate were over 340 $\mathrm{MPa}$ and 2,600 $\mathrm{MPa}$, respectively.

\section{Acknowledgment}

This study was part of the "Leaders in INdustry-university Cooperation (LINC)" Project, supported by the Ministry of Education, Science \& Technology (MEST) and the National Research Foundation of Korea (NRF).

\section{REFERENCES}

1. H. K. Lee, K.-S. Cho, M. H. Jang, C. W. Jang, S. M. Kim, and M. Y. Kim, "Characteristics of Large Green and Sin- 
tered Alumina Ceramics by Filter Pressing (in Korean), " J. Kor. Ceram. Soc., 46 [3] 306-12 (2009).

2. A. Kell and P. Blank, "The Influence of Shaping Method on the Grain Size Dependence of Strength in Dense Submicrometer Alumina,” J. Eur. Ceram. Soc., 16 [11] 1189-200 (1996).

3. W. E. Lee and W. M Rainforth, "Shape Forming," pp. 31-33 in Ceramic Microstructures, Property Control by Processing, Chapman \& Hall, London, 1994.

4. R. Janssen, S. Scheppokat, and N. Claussen, "Tailor-made Ceramic-based Components-Advantages by Reactive Processing and Advanced Shaping Techniques," J. Eur. Ceram. Soc., 28 [7] 1369-79 (2008).

5. F. M. Tiller and C.-D. Tsai, "Theory of Filtration of Ceramics: 1, Slip Casting," J. Am. Ceram. Soc., 69 [12] 882-87 (1986).

6. G. Tari, S. M. Olhero, and J. M. F. Ferreira, "Influence of Temperature on the Colloidal Processing of Electrostatically Stabilized Alumina Suspensions,” J. Mater. Process. Tech., 137 [1-3] 102-09 (2003).

7. G. Tari and J. M. F. Ferreira, "Influence of Solid Loading on Drying Shrinkage Behaviour of Slip Cast Bodies," J. Eur. Ceram. Soc., 18 [5] 487-93 (1998).

8. S. C. Danforth and M. Velazques, "Permeability of Colloidally Filtered (Slip Cast) Monosized Ceramic Powders," pp. 239-43 in Mater. Res. Soc. Symp. Proc. 24, Eds. by J. H. Crawford, Jr, Y. Chen, and W. A. Sibley, Elsevier, New York, 1984.

9. T. Tambas, Z. E. Erkmen, and S. Özgen, "Improvement on the Slip Casting of Bayer-Processed Alumina," Am. Ceram. Soc. Bull., 86 [2] 9201-06 (2007).

10. F. F. Lange and K. T. Miller, "Pressure Filtration: Consolidation Kinetics and Mechanics," Am. Ceram. Soc. Bull., 66 [10] 1498-504 (1987).

11. A. Dietrich, A. Neubrand, and Y. Hirata, "Filtration Behavior of Nanoparticulate $\mathrm{CeO}_{2}$ Slurries," J. Am. Ceram. Soc., 85 [11] 2719-24 (2002).

12. Y. Hirata, K. Onoue, and Y. Tanaka, "Effects of $\mathrm{pH}$ and Concentration of Aqueous Alumina Suspension on Pressure Filtration Rate and Green Microstructure of Consolidated Powder Cake," J. Ceram. Soc. Jpn., 111 [2] 93-99 (2003).

13. Y. Hirata, M. Nakamura, M. Miyamoto, Y. Tanaka, and X. H. Wang, "Colloidal Consolidation of Ceramic Nanoparticles by Pressure Filtration," J. Am. Ceram. Soc., 89 [6] 1883-89 (2006).

14. S. Raha, K. C. Khilar, P. C. Kapur, and Pradip, "Regularities in Pressure Filtration of Fine and Colloidal Suspensions," Int. J. Miner. Process., 84 [1-4] 348-60 (2007).

15. Y. Hirataw and Y. Tanaka, "Pressure Filtration Model of Ceramic Nanoparticles,” J. Am. Ceram. Soc., 91 [3] 819-24 (2008).

16. K.-S. Cho, J. Kim, S. Y. Lee, J. P. Oh, and S. I. Park, "Shape Forming of Alumina by Step Pressure-Vacuum Hybrid Slip Casting," Appl. Mech. Mater., 217-219 1899902 (2012).
17. K.-S. Cho, I.-B. Song, and J. Kim, "Homogeneous Shape Forming of Alumina by Pressure-Vacuum Hybrid Slip Casting (in Korean)," J. Kor. Ceram. Soc., 49 [6] 592-600 (2012).

18. K.-S. Cho, H-K. Lee, and B.-J. Woo, "Effects of Step Pressure on the Shape Forming of Alumina by Pressure-Vacuum Hybrid Slip Casting (in Korean)," J. Kor. Ceram. Soc., 50 [2] 142-48 (2013).

19. A. Aksay and C. H. Schilling, "Colloidal Filtration Route to Uniform Microstructure," pp. 483-91 in Ultrastructure Processing of Ceramics, Glasses, and Composites. Eds. by L. L. Hench, and D. R. Ulrich. Wiley, New York, 1984.

20. E. G. Blanchard, "Pressure Casting Improves Productivity," Am. Ceram. Soc. Bull., 67 [10] 1680-83(1988).

21. T. Uchikoshi, Y. Sakka, K. Ozawa, and K. Hiraga, "Pressure Filtration and Sintering of Fine Zirconia Powder," J. Eur. Ceram. Soc., 18 [6] 669-74 (1998).

22. K. Kendall and M. R. Kosseva, "Nanoparticle Aggregation Influenced by Magnetic Fields,” Colloids Surf., A, 286 [1-3] 112-16 (2006).

23. K. Matsushima, Y. Hirata, N. Matsunaga, and S. Sameshima, "Pressure Filtration of Alumina Suspensions under Alternating Current Field," Colloids Surf., A, 364 [1-3] 138-44 (2010).

24. ASTM C 773-88, "Standard Test Method for Compressive (Crushing) Strength of Fired Whiteware Materials," American Society for Testing and Materials, West Conshohocken, PA, 1999.

25. C.-W. Hong, "New Concept for Simulating Particle Packing in Colloidal Forming Processes," J. Am. Ceram. Soc., 80 [10] 2517-24 (1997).

26. J. D. Yates and S. J. Lombado, "Effect of Solid Loading and Dispersant Concentration on Strain Mismatch and Deformation of Slip-cast Green Bodies," J. Am. Ceram. Soc., 84 [10] 2274-80 (2001).

27. G. W. Scherer, "Theory of Drying," J. Am. Ceram. Soc., 73 [1] 3-14 (1990).

28. J. L. Amoros, E. Sanchez, V. Cantavella, and J. C. Jarque, "Evolution of the Mechanical Strength of Industrially Dried Ceramic Tiles during Storage," J. Eur. Ceram. Soc., 23 [6] 1839-45 (2003).

29. J. D. Yates and S. J. Lombado, "The Effect of Plaster Composition and Binder Concentration on Strain Mismatch and Deformation of Slip-Cast Green Bodies," Mater. Sci. Eng., A, 337 [1-2] 297-305 (2002).

30. L. Curkovic and M. F. Jelaca, "Dissolution of Alumina Ceramics in HCl Aqueous Solution," Ceram. Int., 35 [5] 2041-45 (2009).

31. R. B. Heimann, "Oxide Ceramics: Structure, Technology, and Application," pp. 175-252 in Classic and Advanced Ceramics, Wiley-VCH Verlag GmbH \& Co., Weinheim, 2010.

32. W. E. Lee and W. M Rainforth, "Structural Oxides 1: $\mathrm{Al}_{2} \mathrm{O}_{3}$ and Mullite," pp. 255-316 in Ceramic Microstructures Property Control by Processing, Chapman \& Hall, London, 1994. 